\title{
Aus der ärztlichen Werkstatt
}

Erhard Taverna

Im Zuge Magrittes

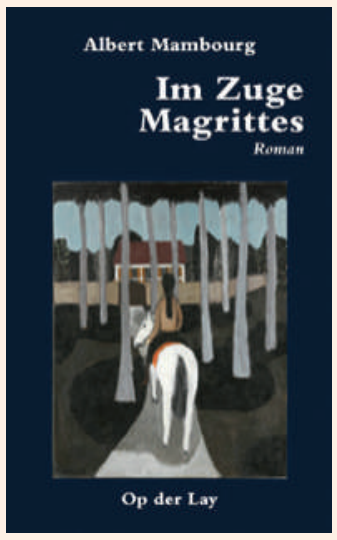

Albert Mambourg Im Zuge Magrittes

Esch/Lauer, Luxemburg Op der Lay; 2011 204 Seiten. 15.90 Euro. ISBN 978-2-87967-176-5

Der Name des Romanhelden ist nicht zufällig der des belgischen Surrealisten Magritte. Man kann das Buch als erotisch aufgeladene Gesellschaftssatire lesen, in der betrogene Ehemänner, üppige Leichenschmäuse und ein sehr vitaler Kleinstadt-Don-Juan ein groteskes Theaterstück aufführen. Es wird stets opulent aufgetragen, von den Rezepten des Sternekochs im Bären zu den ausschweifenden Vereinigungen in fremden Schlaf- und Badezimmern bis zu der sexuellen Aufklärung eines Bischofs mit Hilfe mittelalterlicher Tafeln zur weiblichen Anatomie. Magritte ist ein belesener Schwadroneur, dessen ausufernde Rhetorik akademisch gebildete Gegner spielend schachmatt setzt. Ein Hofnarr mit einem zangenbedingten Geburtsschaden, denn: «Magritte war ein reiner Triebmensch, er zeigte also keine Scham, er hatte kein Ehrgefühl und keine Manieren, weil ihm der züchtigende Eingriff des Vorderhirns, der Seele also, fehlte.» Der Regisseur dieser bunten Truppe von Juristen, Ärzten und Klerikern war jahrzehntelang Gynäkologe in Luzern. Vielleicht nimmt er auch uns Leser, wie der berühmte Maler, auf den Arm. Diesem zufolge sind Bilder eine vertrackte Sache, denn: «Kein Gegenstand ist mit seinemNamen den könnte, der besser zu ihm passt.» Der Magritte im Buch hatte viele Anhänger: «Mit seiner Diskussionsmethode - Plattitüden, gemischt mit grosser Philosophie - hatte er einen rechten Erfolg. Eine kleine, neue Religion war entstanden.»

\section{Blutspurlos}

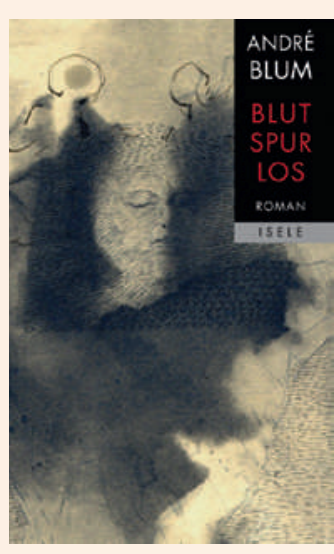

André Blum Blutspurlos

Kreuzlingen: Edition Isele; 2012.

232 Seiten. $25.90 \mathrm{CHF}$ ISBN 928-3-86142-541-0

Ein Kardiologe verlässt aufgeschreckt sein Labor. Er fährt fluchtartig in die französische Abgeschiedenheit der Tortue. Im Landhaus eines Freundes will er sich seinem Feind stellen, planmässig, nach einem strikten Schema, mit äusserster Disziplin. Er macht sich zum Objekt eines wissenschaftlichen Experimentes, will seine Strategie tabellarisch auf dem Computer festhalten, nur Fakten notieren, sich selbst in der Anlage eines Kriegstagebuchs subtrahieren. Nichts entgeht seinem Misstrauen, nachts hört er bedrohliche Geräusche. Zwanghaft und zunehmend paranoid, treibt es ihn unaufhaltsam der psychotischen Katastrophe zu. Das wird alles beklemmend, mit grosser Präzision und gutformuliert beschrieben. Aus den Briefen an die verstorbene Ehefrau entwickelt der Autor die schwierige Beziehung zwischen einer sensiblen Poetin und einem überrationalen, gefühlsgestörten Mann, der aufgrund seiner tragischen Biografie überzeugt ist, einen Todeskeim in sich zu tragen. Dieser halluziniert, hört Stimmen in seinem Kopf, bekommt von der Nachbarin ein Jagdgewehr ausgeliehen, verletzt sich und stirbt fast an einer Lungenentzündung. Schlimmer kann es nicht mehr werden. Da betritt er widerwillig eine Kapelle, deren Reliquie, eine Mumie, sein Freund untersuchen soll. Der Ich-Erzähler, Schrittmacherspezialist und ElektronikIngenieur soll ihm dabei helfen. Sind es die Gedichte der verstorbenen Ehefrau, ist es die Heilige, deren Existenz höchst zweifelhaft erscheint, die Geschichte nimmt eine überraschende Wendung. André Blum hat in seinem Erstling geschickt und sprachmächtig 
eine Krankheits- und Liebesgeschichte mit archäologischem Gespür zu einer Heilsgeschichte mit einem offenen Ausgang verflochten.

\section{Pepino der kleine Esel}

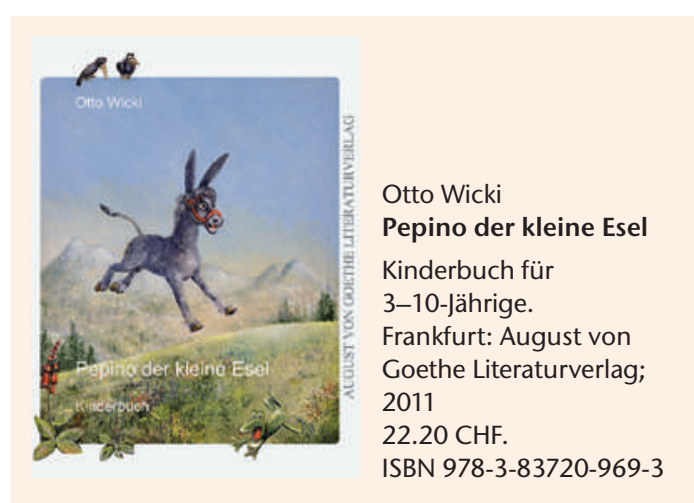

Auf der Website von Otto Wick steht sein Eingangsmotto: «Am Ende des Lebens bleibt Dir nur das, was Du anderen geschenkt hast.» Der 1932 geborene Schriftsteller hat schon vieles geschenkt, als Chirurg war er Co-Autor und Begründer der «Medizinischen Checklisten», einer flexiblen Taschenbuchreihe im Thieme-Verlag. Es folgten zahlreiche Erzählungen und jetzt auch ein Kinderbuch mit 15 schönen Farbbildern von Bernd Günther. Pepino muss schwere Lasten tragen, immer angetrieben von Marco, dem Ziegenhirten. Im Traum sieht Marco, wie der hungernde Esel dem fast erblindeten Fuchs seine Augen im Austausch für ein fettes Huhn ausleiht, das er Marco gegen viel Heu überlässt. Beide werden bei diesem Handel so dick, dass sie nicht mehr für die Arbeit taugen. Von da an behandelt Marco seinen Esel liebevoller. Pepino bekommt täglich frisches Heu und saftige Karotten. Das Hungern hat ein Ende, und sie arbeiteten glücklich und als Freunde bis zum heutigen Tag. Denn, so die Moral der Geschichte: «Wer Gutes tut, bekommt alles doppelt zurück.»

\section{Begegnung mit dem Fremden}

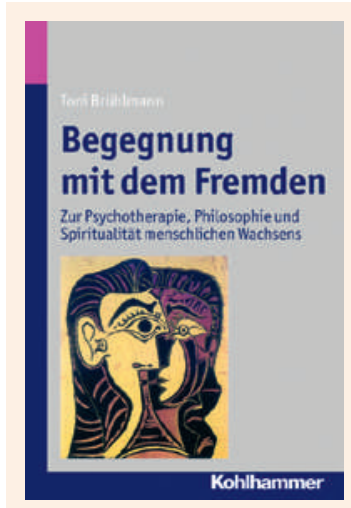

Toni Brühlmann Begegnung mit dem Fremden

Stuttgart: Kohlhammer; 2011

178 Seiten. $44.90 \mathrm{CH}$ ISBN 978-3-17021-858-1

Toni Brühlmann, Psychiater, Verfasser zahlreicher Publikationen zu Burn-out, Depression und narzisstischen Störungen, versieht sein Fachbuch mit dem
Untertitel: Zur Psychotherapie, Philosophie und Spiritualität menschlichen Wachsens. Sein Werk hat er in sechs Kapitel unterteilt: Die Voraussetzungen menschlichen Wachsens; der historische Kontext: die heutige Krisenzeit; Anthropologie in psychotherapeutischer Sicht; Anthropologie in philosophischer Sicht; Therapie als Emanzipation und Resignation und im Anhang: postnarzisstische Übergangszeit - quo vadis? Das fundiert und konzentriert geschriebene Buch richtet sich in erster Linie an Fachpersonen aus der Psychotherapie, an Personen geisteswissenschaftlicher Richtungen und an interessierte Laien. Seine besondere Aufmerksamkeit gilt dem fortschreitenden Irrationalismus der Gegenwart und den Auswüchsen und Schäden der Leistungs- und Erfolgskultur mit ihrer Subjektzentrierung. Zusammenfassungen am Ende eines jeden Kapitels bringen die anspruchsvolle Lektüre auf den Punkt. Brühlmann beschreibt die bisherigen Denkwege als eine Philosophie der Abwesenheit, als Philosophie der Verneinung und Exteriorität, da das Bestimmende im «Ausserhalb» gesehen wird. Er wünscht sich eine zeitgemässere Philosophie der Anwesenheit, die aus der Verbindung, Bejahung und Liebe, entgegen der heutigen Realität, hervorgehen würde. Das anspruchsvolle Buch kann als konzises Kompendium der Philosophie und Ethik, als Lehrbuch der Psychotherapie und als kritisches Essay gelesen werden, das mit den Worten des Autors «über die Grenze und die heutige Borderline-Ära einen Blick hinüber werfen will».

\section{Operationstechniken in der Fusschirurgie}

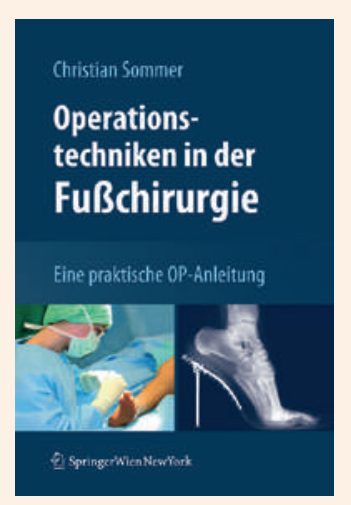

Christian Sommer Operationstechniken in der Fusschirurgie Heidelberg: Springer-Verlag KG; 2012 350 Seiten. $319 \mathrm{Abb}$ $119.00 \mathrm{CHF}$ 978-3-21199-422-1

Der Autor Dr. med. Christian Sommer ist Facharzt für Orthopädische Chirurgie und Traumatologie. Sein Spektrum umfasst korrektive Fussoperationen, Revisions- und Rheumachirurgie und Prothetik. Seine praktische OP-Anleitung versteht sich als Orientierungshilfe aus der Praxis für die Praxis. Der vorliegende Band ergänzt das bereits in zweiter Auflage bestehende Buch über die Grundlagen der Fusschirurgie. 319 sehr schöne Farbabbildungen weisen den Weg durch die Grundlagen- und Spezialkapitel. Der Autor hat seine Sprache genauso im Griff wie sein Skalpell. Knapp, klar und gut ver- 
ständlich schildert er die Voraussetzungen des Operierens, die Nachbehandlung und die zahlreichen, komplexen Pathologien des Fusses. Literaturempfehlungen, eine kurze Anleitung zum Gebrauch des Hallux-Goniometers und ein ausführliches Sachverzeichnis beenden die auch für Nichtspezialisten lesenswerten Ausführungen. Statistiken belegen, dass fast die Hälfte der Patienten den
Orthopäden wegen Fussproblemen aufsuchen. Fehlstellungen, Überlastungssymptome, Instabilitätsprobleme, Arthrosen und Sportunfälle sind auch in der hausärztlichen Praxis häufige Konsultationsgründe. Es gibt also gute Gründe, genauer zu wissen, wie ein renommierter Kollege in seiner Privatpraxis damit umgeht. Empfohlen sei auch die interessante Website www.fussclinic.ch 